\title{
Incidental Detection of Cyclospora cayetanensis during General Health Screening: A Case Study from Singapore
}

\author{
Jean-Marc Chavatte ${ }^{1^{*}}$ and Roland Jureen ${ }^{2}$
}

${ }^{1}$ Malaria Reference Centre, National Public Health Laboratory, Ministry of Health, Singapore

${ }^{2}$ Department of Laboratory Medicine, National University Hospital, Singapore

*Corresponding author: Jean-Marc Chavatte, National Public Health Laboratory, Ministry of Health, 3 Biopolis Drive, Singapore, Tel: (65) 6837 8311; E-mail: jeanmarc_chavatte@moh.gov.sg

Received date: November 10, 2016; Accepted date: November 19, 2016; Published date: November 30, 2016

Copyright: (C) 2016 Chavatte JM, et al. This is an open-access article distributed under the terms of the Creative Commons Attribution License, which permits unrestricted use, distribution, and reproduction in any medium, provided the original author and source are credited.

\begin{abstract}
Background: Cyclospora cayetanensis is a coccidian parasite recently recognized and characterized that cause enteritic infection worldwide. Endemic in tropical and subtropical countries where asymptomatic carriage is common, this parasite is also frequently associated with foodborne and waterborne outbreaks and reported from travellers in industrialized countries. Poorly characterized by routine laboratory procedures, the oocysts of $C$. cayetanensis are also discontinuously shed, altogether making this parasite difficult to detect. In Singapore, information about $C$. cayetanensis are scarce and the endemicity status is unknown, while the country seems vulnerable to outbreak occurrence.
\end{abstract}

Case study: The present report describes the incidental laboratory finding of $C$. cayetanensis oocysts in the stool of an asymptomatic immunocompetent patient attending a general health screening. Initial suspicion about the diagnosis was confirmed by several morphological methods and concurrently by amplification and sequencing of the parasite DNA. Along the study, co-infections with Blastocytis sp. ST3, Cryptosporidium parvum/hominis and the nonpathogenic Entamoeba hartmanni were noticed and confirmed by molecular methods.

Conclusions: This report could serve to raise awareness about Cyclospora cayetanensis and the asymsptomatic carriage of enteritic protozoa, and as a reminder about the risk of cyclosporiasis especially in the most susceptible populations such as: children, elderly and immunocompromised.

Keywords: Cyclospora cayetanensis, Entamoeba hartmanni; Blastocystis sp. ST3; Incidental detection; Asymptomatic carriage; Health screening; Case report

\section{Introduction}

Cyclospora cayetanensis is a Protozoan parasite of the human enteric tract only recently characterized and described $[1,2]$ and now recognized as an important emerging cause of diarrhoea worldwide [3]. Probably first associated with human illness by [4] who reported an undescribed coccidian parasite related to Isospora, the identity of this pathogen remained controversial until 1993 when [1] managed to induce the sporulation of this organism and demonstrated that it has two sporocysts each containing two sporozoites and therefore it was an oocyst of a coccidian parasite related to the genus Cyclospora and proposed the name Cyclospora cayetanensis Ortega, Gilman \& Sterling, 1994 [2]. Before, it might have been overlooked as Blastocystis [5], then subsequently considered as a coccidian parasite [6,7], a spore of fungus [6], an algae-like organism related to the blue-green algae $[8,9]$ and was therefore also called cyanobacterium-like organism, abbreviated CLB [10-13]. Even after being identified as Cyclospora, it has remained reported as CLB for "coccidian/cyanobacterium-like body" $[14,15]$ and sometime also for "cyclospora like body" [13]. Later, phylogenetic analysis has confirmed that $C$. cayetanensis truly is a coccidian parasite closely related to the avian Eimeria species [16].
C. cayetanensis is widely distributed worldwide but seems more common in tropical and subtropical regions [17]. In developing countries, it is regularly detected among local populations in both symptomatic and asymptomatic adults and children [17-19] and infections have been linked to different sources such as contaminated water, food and soil and also contact with animals, sanitation and low socioeconomic status [17]. In developed countries, it is usually detected in travellers returning from tropical and subtropical regions [20-22]; and it is also a recurrent cause of foodborne outbreaks related to consumption of imported raw fruits and vegetables in North America since the 1990s $[21,23,24]$. Frequently detected in water for human consumption in endemic countries [25,26], waterborne outbreaks have been reported in US [27] and in Nepal even after water chlorination [28].

As a major emerging cause of diarrhoea outbreaks, cyclosporiasis is a notifiable disease in US [21]. In Singapore, the Ministry of Health $(\mathrm{MOH})$ doesn't collect information about this pathogen that is rarely reported (e.g. only 4 cases detected at National University Hospital in the last 4 years). To the authors knowledge, in the literature, Singapore was only listed as a visited country along with Indonesia in the first report of imported cyclosporiasis in Germany [29] and was also mentioned along with Malaysia as suspected port of embarkation of contaminated food potentially implicated in a cruise cyclosporiasis outbreak [30]. 
The present report describes the incidental laboratory finding of $C$. cayetanensis in the stool samples of a healthy, asymptomatic patient participating into a general health screening.

\section{Case Study}

Patient: A 35 years old immunocompetent Indonesian male without any symptoms presented at the National University Hospital (NUH) for a general health screening. The physical examination was unremarkable and there were no complaints of gastrointestinal disturbance noted. As part of the health screening a stool specimen was collected for screening for ova, cysts and parasites.

NUH laboratory: Initially, a direct examination by saline wet mounts was made by mixing a small volume of stool sample with a drop of physiological saline on a glass microscope slide according to standard procedure [31]. With light microscope at low (100X) then medium (400X) magnification some unidentified cysts like bodies were noted.

Thin faecal smears were made and fixed in methanol prior permanent staining by Kinyoun acid-fast and modified trichrome stains respectively following standard procedures [31]. The smears were observed with light microscope at medium (400X) then high (1000X) magnification.

They revealed cysts of $8-10 \mu \mathrm{m}$, totally unstained in trichrome (Figures 1A-1C) and only partly acid-fast with some cysts stained in light to dark pink while the wide majority remained unstained (Figures $1 \mathrm{D}-1 \mathrm{~F})$. This result was suggestive of $C$. cayetanensis. The original smears and two aliquots of the faecal material in sodium acetate-acetic acid-formalin (SAF) and absolute ethanol respectively were passed to the National Public Health Laboratory (NPHL) for confirmation.

NPHL microscopy: Morphological observation was performed with an Olympus CX31 microscope and microphotographs were taken with a Nikon Eclipse 80i microscope equipped with a Nikon DS Ril camera and the Elements-D Imaging Software at medium (400X) and high (1000X) magnification. The original smears were confirmed positive for C. cayetanensis (Figures 1A-1F).

Additional thin faecal smears were prepared from the SAF fixed sample for UV fluorescence microscopy and safranin staining tests. The safranin stained smears were prepared according to [32] procedure with microwave heating and showed a uniform staining of all the oocysts (Figures 1G-1I).

Under UV light, the oocysts were autofluorescent even after fixation, and displayed a typical blue colour with UV excitation set at 330-365 nm (Figures 2A-2D) and green colour with UV excitation set at $450-490 \mathrm{~nm}$ (Figures 2B-2E). The safranin being a fluorophore itself, the oocysts stained with this dye were also visible under UV light and displayed a red fluorescence with excitation set at 528-552 $\mathrm{nm}$ (Figures 2C-2F).

Taken all together these results confirmed the nature of the organism as oocyst of $C$. cayetanensis. Additional findings were made on the trichrome stained smear with the detection of numerous Entamoeba cysts morphologically compatible with Entamoeba hartmanni (Figure 1J), some Blastocystis sp. cysts (Figure 1K) and some rare oocysts suggestive of Cryptosporidium spp. (Figure 1L).

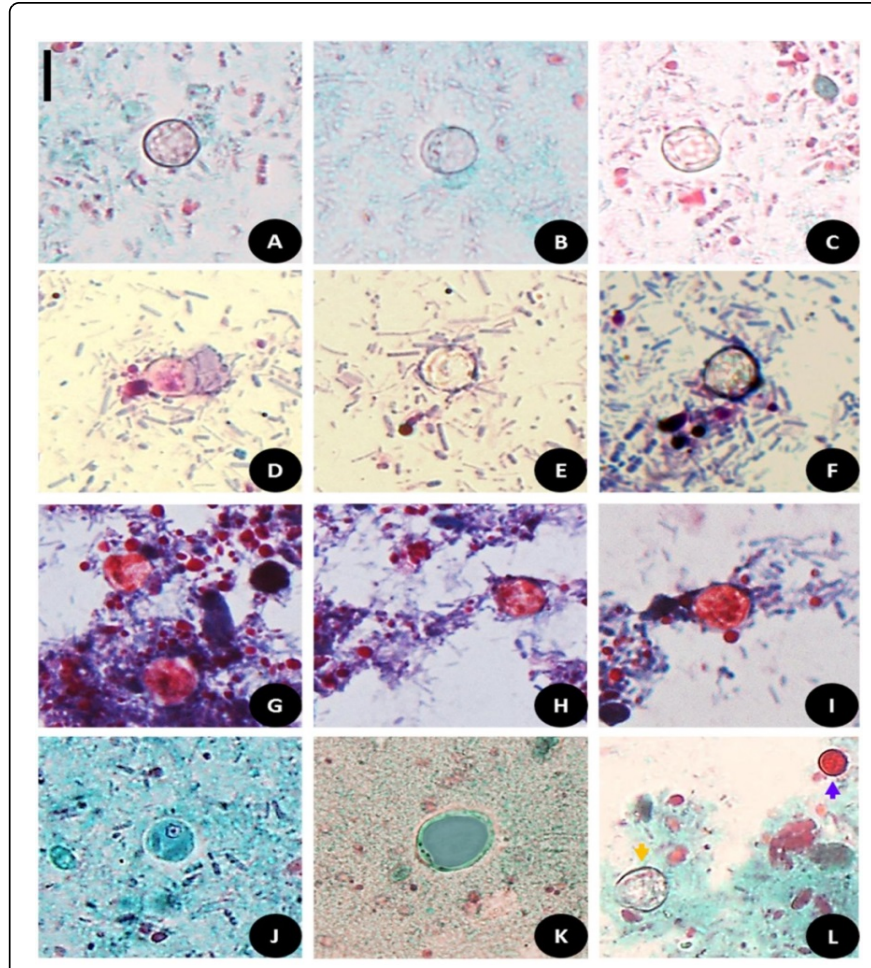

Figure 1: Microphotographs of enteric Protozoa detected from faecal smears with different staining methods. A-C, L (orange arrow): oocysts of $C$. cayetanensis in trichrome stain, typically unstained; D-F: oocysts of $C$. cayetanensis in acid-fast stain, inconsistently stained (D) or unstained (E,F); G-I oocysts of $C$. cayetanensis in safranin stain, uniformly stained; J: cyst of Entamoeba hartmanni in trichrome stain; K: cyst of Blastocystis sp. in trichrome stain; L (purple arrow): oocyst of Cryptosporidium parvum/hominis in trichrome stain. Scale bar for all pictures $=10$ $\mu \mathrm{m}$.

Molecular methods: Molecular tests for Cyclospora, Entamoeba, Blastocystis and Cryptosporidum detection were initiated from the ethanol preserved stool sample.

DNA extraction was performed with the QIAmp ${ }^{\circ}$ DNA stool mini kit (Qiagen ${ }^{\circ}$ after 10 freeze/thaw cycles $\left(1 \mathrm{~min}-196^{\circ} \mathrm{C}\right.$ and $1 \mathrm{~min}$ $100^{\circ} \mathrm{C}$ ). DNA was eluted in $\mathrm{AE}$ buffer and stored at $-30^{\circ} \mathrm{C}$. DNA amplifications for each organism were performed by nested-PCR protocols targeting the $18 \mathrm{~S}$ ribosomal RNA (18S rRNA) gene.

The first reaction was common to all organisms and used a pair of $18 \mathrm{~S}$ rRNA universal oligonucleotide primers as described by [33].

For the second amplifications four separate specific reactions were performed according to the protocols proposed by [34] (with the primers pair: ExCycF/ExCycR), [35] (with the primers pair: Eg-SSF1/Eg-SS-CR1), [36] (with the primers set B); and [37] (with primers pair: SSU-F2/SSU-R2); for Cyclospora, Entamoeba spp., Blastocystis sp. and Cryptosporidium spp. respectively. 


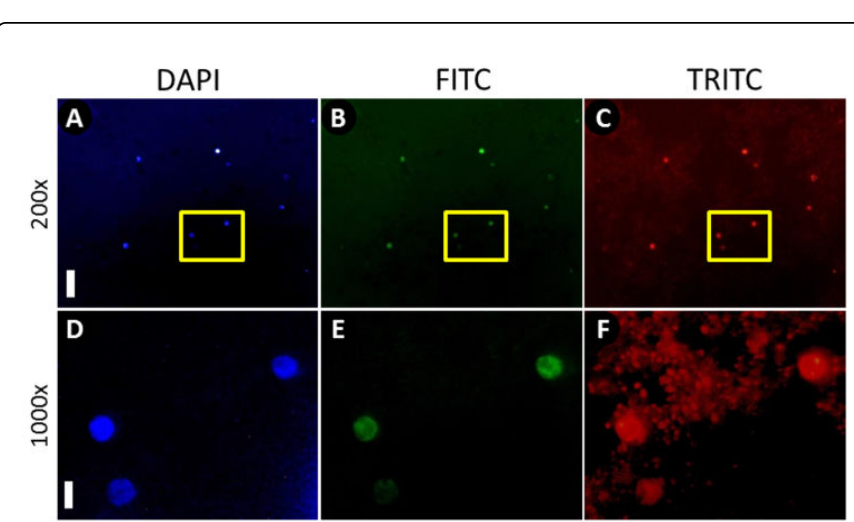

Figure 2: Fluorescence microphotographs of Cyclospora cayetanensis oocysts from faecal smear. A, B: general overview at medium magnification (200X), scale bar $=50 \mu \mathrm{m}$; D-F: detailed view at high magnification (1000X) corresponding to the area represented by the yellow box in medium magnification, scale bar $=10 \mu \mathrm{m} ; \mathrm{A}$, D: autofluorescence of $C$. cayetanensis oocysts under UV light with DAPI filter (EX 340-380 nm, DM $400 \mathrm{~nm}$, BA 435-485 nm); B, E: autofluorescence of $C$. cayetanensis oocysts under UV light with FITC filter (EX 465-495 nm, DM 505 nm, BA 515-555 nm); C,F: fluorescence of safranin stained oocysts with TRITC filter (EX 540/25 nm, DM 565 nm, BA 605/55 nm).

All the PCRs reactions were run on Veriti ${ }^{\oplus}$ Thermal Cycler (Applied Biosystems $s^{\odot}$, in a total volume of $20 \mu \mathrm{L}$ containing $2 \mu \mathrm{L}$ of original DNA template for the first reaction and $1 \mu \mathrm{L}$ of the PCR product of the first reaction as template for each second reaction.

All PCR products were visualised by electrophoresis on QIAxcel $^{\circ}$ Advanced instrument (Qiagen ${ }^{\circledR}$ ) prior purification by the QIAquick ${ }^{\star}$ PCR Purification Kit (Qiagen ${ }^{\circ}$ ); eluted in $30 \mu \mathrm{L}$ of EB buffer; frozen at $-30^{\circ} \mathrm{C}$; then prepared for sequencing in both directions using the BigDye ${ }^{\oplus}$ Terminator v3.1 cycle sequencing Kit (Applied Biosystems ${ }^{\oplus}$ ) and the respective oligonucleotide primers. The BigDye ${ }^{\circledR}$ reaction's products were purified using the BigDye ${ }^{\oplus}$ XTerminator $^{\mathrm{m}}$ Purification $^{\circ}$ Kit (Applied Biosystems $s^{\oplus}$ ) before being sequenced on a 3500xl Genetic Analyzer (Applied Biosystems ${ }^{\oplus}$ ).

For each step, kits and equipment were used according to the manufacturer's recommendations.

Despite multiples attempts the detection of Cryptosporidium spp. remained unsuccessful on the $18 \mathrm{~S}$ rRNA gene.

However Cryptosporidium parvum/hominis DNA was detected in all replicates of a real-time PCR (rt-PCR) assay targeting a gene encoding a DNA-J like protein with high Ct value ( $\overline{\mathrm{x}} 36.45)$. The rtPCR was performed according to the protocol proposed by [38].

Additional rt-PCR assays for Dientamoeba fragilis and Enterocytozoon bieneusi remained negative. All rt-PCRs were performed using the QuantiFast ${ }^{\oplus}$ Probe RT-PCR Kit (Qiagen ${ }^{\circledR}$ ) and run

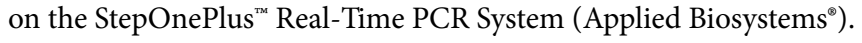

Alignment and cross-checking of the sequences were performed with CLC Main Workbench v7.6 (CLC Bio-Qiagen ${ }^{\circ}$ ) and consensus sequences of $1793 \mathrm{bp}, 1910 \mathrm{bp}$ and $1769 \mathrm{bp}$ representing the nearly complete sequence of the 18S rRNA gene were obtained for Cyclospora, Entamoeba spp. and Blastocystis sp. respectively. There was no sequence obtained for Cryptosporidium parvum/hominis as this organism was only detected by rt-PCR protocol.

Basic Local Alignment Search Tool (BLAST) [39] confirmed the identity of these organisms as Cyclospora cayetanensis, Entamoeba hartmanni and Blastocystis sp. subtype 3 (ST3). The respective sequences were deposited in GenBank under the accession numbers: KX618190 to KX618192.

\section{Discussion}

The present study reports the incidental detection of $C$. cayetanensis in Singapore during the voluntary health screening of a healthy patient. Being asymptomatic the patient didn't take any treatment and was found negative in NUH laboratory during a follow-up appointment two weeks later. This situation highlights the difficulty to detect $C$. cayetanensis oocysts from stool samples because of their intermittent shedding and the low sensitivity of the routine procedures $[17,20]$, while it also highlight the usefulness of special staining procedure, fluorescence microscopy as well as molecular methods.

This Protozoa is now recognized as a major cause of diarrheal illness worldwide that should be investigated especially when virus and bacteria screenings are negative [17,20]. In tropical and subtropical endemic regions [17-22], early and frequent exposure to $C$. cayetanensis seems to be associated with an acquired immunity [40], a decrease of the symptoms [40] with only sometime punctual relapses $[17,21]$. In these regions community based studies show prevalence up to $41.6 \%$ with a high percentage of asymptomatic carriers (62.8\%-98.7\%) [17]. Elsewhere, this coccidian parasite is often epidemic and often reported from travellers [20-22] and also as the cause of waterborne $[27,28]$ and foodborne outbreaks related to imported fresh products [21,23-26].

Similarly to some other industrialized country, C. cayetanensis remains rarely reported and largely understudied in Singapore. It might due to a lack of awareness and/or the difficulty to detect this pathogen [41]. This situation seem surprising as Singapore, is geographically located in a favourable region for the environmental maturation of $C$. cayetanensis oocysts, is surrendered by endemic countries that supply the majority of its food and water; and is visited by hundreds of migrants such as workers, tourists and travellers coming or returning from these endemic countries. For all these reasons Singapore seems to be a vulnerable country for the occurrence of cyclosporiasis outbreaks.

As the prevalence of $C$. cayetanensis in diarrheic patients as well as in in susceptible populations such as the children, elderly, travellers and immunocompromised in Singapore, is unknown the endemicity of the infection for the nation is unknown and could only be partly reflected through the report and study of sporadic cases among local residents and tourists visiting the country. In the present case study, it was not possible to confidently identify the origin of the infection as the patient originated from Indonesia which is an endemic country $[15,20,29,41-43]$ and there was no information about a past onset of symptoms.

Given the particularities in the biological cycles of the different parasitic protozoa detected, it is likely that the patient had multiple exposures to faecally contaminated water and/or food. The present report about an asymptomatic carrier of multiple enteritic protozoa should serve as a reminder that introduction and dissemination of these organisms could possibly lead to contamination of soil, water 
sources and perhaps even food especially for $C$. cayetanensis as its oocysts are resistant to basic food and water treatments $[20,27,28]$.

This report should also raise awareness locally to all clinicians, medical practitioners, laboratory staffs and public health officers about C. cayetanensis. Until now, cyclosporiasis outbreaks in Singapore could have been overlooked or really avoided by the strict control of food and water quality. In the future, in the perspective of an increase of both the population and its inherent needs of importation of fresh products, $C$. cayetanensis could potentially become a public health problem in Singapore. To assess the risk and monitor the situation, it would be desirable to evaluate the endemicity status in both symptomatic and asymptomatic individuals.

\section{Acknowledgments}

The authors are grateful to Ms Sharain Binte Abdul Kadir from NUH Department of Laboratory Medicine who first suspected some unusual cysts during Ova-Cyst-Parasite procedure and to A/Prof Lin RTPV who agreed and supported this study. The study was funded by the NPHL of the MOH.

\section{Author's contributions}

RJ discovered the case and proposed its study. JMC confirmed the case by morphological methods, performed the molecular studies and wrote the manuscript. Both authors read and approved the final manuscript.

\section{Conflict of interest}

The authors declare that they have no conflict of interest.

\section{References}

1. Ortega YR, Sterling CR, Gilman RH, Cama VA, Díaz F (1993) Cyclospora species: a new protozoan pathogen of humans. N Engl J Med 328: 1308-1312.

2. Ortega YR, Gilman RH, Sterling CR (1994) A new coccidian parasite from humans. J Parasitol 80: 625-629.

3. Chacín BL (2012) Cyclospora cayetanensis as a global health problem. Epidemiol 2: 3.

4. Ashford RW (1979) Occurrence of an undescribed coccidian in man in Papua New Guinea. Ann Trop Med Parasitol 73: 497-500.

5. Babcock D, Houston R, Kumaki D, Shlim D (1985) Blastocystis hominis in Kathmandu, Nepal. N Engl J Med 313: 1419.

6. Soave R, Dubey JP, Ramos LJ, Tummings M (1986) A new intestinal pathogen? Clin Res 34: 533.

7. Naranjo J, Sterling C, Gilman R (1989) Cryptosporidium muris like objects from fecal samples of Peruvians. Program and abstracts of the 38th Annual Meeting of the American Society of Tropical Medicine and Hygiene (Honolulu). p. 243.

8. Long EG, Ebrahimzadeh A, White EH, Swisher B, Callaway CS (1990) Alga associated with diarrhea in patients with acquired immunodeficiency syndrome and in travellers. J Clin Microbiol 28: 1101-1104.

9. Shlim DR, Cohen MT, Eaton M, Rajah R, Long EG, et al. (1991) An alga like organism associated with an outbreak of prolonged diarrohea among foreigners in Nepal. Am J Trop Med Hyg 45: 383-389.

10. Centers for Disease Control and Prevention (1991) Outbreaks of diarrheal illness associated with cyanobacteria (blue-green algae) like bodies: Chicago and Nepal, 1989 and 1990. MMWR Morb Mortal Wkly Rep 40: 325-327.
11. Long EG, White EH, Carmichael WW, Quinlisk PM, Raja R, et al. (1991) Morphologic and staining characteristics of a cyanobacterium-like organism associated with diarrhea. J Infect Dis 164: 199-202.

12. Pollock RGC, Bendall RP, Moody A, Chiodini PL, Churchill DR (1992) Traveller's diarrhoea associated with cyanobacterium-like bodies. Lancet 340: 556-557.

13. Bendall RP, Lucas S, Moody A, Tovey G, Chiodini PL (1993) Diarrhoea associated with cyanobacterium-like bodies: a new coccidian enteritis of man. Lancet 341: 590-592.

14. Hoge CW, Shlim DR, Rajah R, Triplett J, Shear M, et al. (1993) Epidemiology of diarrhoeal illness associated with coccidian-like organism among travellers and foreign residents in Nepal. Lancet 341:1175-1179.

15. Butcher AR, Lumb R, Coulter E, Nielsen DJ (1994) Coccidian/ cyanobacterium-like body associated diarrhea in an Australian traveller returning from overseas. Pathology 26:59-61.

16. Relman DA, Schmidt TM, Gajadhar A, Sogin M, Cross J, et al. (1996) Molecular phylogenetic analysis of Cyclospora, the human intestinal pathogen, suggests that it is closely related to Eimeria species. J Infect Dis 173:440-445.

17. Chacín-Bonilla L (2010) Epidemiology of Cyclospora cayetanensis: A review focusing in endemic areas. Acta Trop 115:181-193.

18. Thima K, Mori H, Praevanit R, Mongkhonmu S, Waikagul J, et al. (2014) Recovery of Cyclospora cayetanensis among asymptomatic rural Thai school children. Asian Pac J Trop Med 7:119-123.

19. Bhandari D, Tandukar S, Parajuli H, Thapa P, Chaudhary P, et al. (2015) Cyclospora Infection among school children in Kathmandu, Nepal: Prevalence and associated risk factors. Trop Med Health 43:211-216.

20. Ortega YR, Sanchez R (2010) Update on Cyclospora cayetanensis, a foodborne and water-borne parasite. Clin Microbiol Rev 23: 218-234.

21. Hall RI, Jones JL, Herwaldt BL (2011) Surveliance for laboratoryconfirmed sporadic cases of Cyclosporiasis United States. Morb Mortal Wkly Rep 60:1-11.

22. Nichols GL, Freedman J, Pollock KG, Rumble C, Chalmers RM, et al. (2015) Cyclospora infection linked to travel to Mexico, June to September 2015. Euro Surveill 20:30048.

23. Herwaldt B (2000) Cyclospora cayetanensis: A Review, focusing on the outbreaks of Cyclosporiasis in the 1990s. Clin Inf Dis 31: 1040-1057.

24. Buss BF, Joshi MV, Dement JL, Cantu V, Safranek TJ (2016) Multistate product traceforward investigation to link imported romaine lettuce to a US cyclosporiasis outbreak - Nebraska, Texas, and Florida, June-August 2013. Epidemiol Infect 144: 2709-2718.

25. Dowd SE, John D, Eliopolus J, Gerba CP, Naranjo J, et al. (2003) Confirmed detection of Cyclospora cayetanensis, Encephalitozoon intestinalis and Cryptosporidium parvum in water used for drinking. J Water Health 1: 117-123.

26. Kwakye NG, Borketey P, Mensah AI, Asmah R, Ayeh KP (2007) Sachet drinking water in Accra: the potential threats of transmission of enteric pathogenic protozoan organisms. Ghana Med J 41: 62-67.

27. Huang P, Weber JT, Sosin DM, Griffin PM, Long EG, et al. (1995) The first reported outbreak of diarrheal illness associated with Cyclospora in the United States. Ann Intern Med 123: 409-414.

28. Rabold JG, Hoge CW, Shlim DR, Kefford C, Rajah R, et al. (1994) Cyclospora outbreak associated with chlorinated drinking water. Lancet 344: 1360-1361.

29. Petry F, Hofstätter J, Schulz BK, Deitrich G, Jung M, et al. (1997) Cyclospora cayetanensis: first imported infections in Germany. Infection 25: $167-170$.

30. Gibb RA, Nanyonjo R, Pingaut NM, Combs BG, Mazzucchelli T, et al. (2013) An outbreak of Cyclospora infection on a cruise ship. Epidemiol Inf 141: 508-516.

31. Garcia LS (2010) Parasitology. Clinical Microbiology Procedures Handbook - Vol. 2 (3rd edn.) ASM Press, Washington, DC. 
Citation: Chavatte JM, Jureen R (2016) Incidental Detection of Cyclospora cayetanensis during General Health Screening: A Case Study from Singapore. J Trop Dis 4: 224. doi:10.4172/2329-891X.1000224

Page 5 of 5

32. Visvesvara GS, Moura H, Kovacs-Nace E, Wallace S, Eberhard ML (1997) Uniform staining of Cyclospora oocysts in fecal smears by a modified safranin technique with microwave heating. J Clin Microbiol 35: 730-733.

33. Medlin L, Elwood HJ, Sickel S, Sogin ML (1988) The characterization of enzymatically amplified eukaryotic 16S-like rRNA-coding regions. Gene 71: 491-499.

34. Li G, Xiao S, Zhou R, Wadeh H (2007) Molecular characterization of Cyclospora-like organism from dairy cattle. Parasitol Res 100: 955-961.

35. Foo PC, Chan YY, See Too WC, Tan ZN, Wong WK, et al. (2012) Development of a thermostabilized, one-step, nested, tetraplex PCR assay for simultaneous identification and differentiation of Entamoeba species, Entamoeba histolytica and Entamoeba dispar from stool samples. J Med Microbiol 61: 1219-1225.

36. Wong KHS, Ng GC, Lin RTP, Yoshikawa H, Taylor MB, et al. (2008) Predominance of subtype 3 among Blastocystis isolates from a major hospital in Singapore. Parasitol Res 102: 663-670.

37. Xiao L, Escalante L, Yang C, Sulaiman I, Escalante AA, et al. (1999) Phylogenetic analysis of Cryptosporidium parasites based on the smallsubunit rRNA gene locus. Appl Environ Microbiol 65: 1578-1583.
38. Bruijnesteijn van Coppenraet LES, Wallinga JA, Ruijs GJHM, Bruins MJ, Verweij JJ (2009) Parasitological diagnosis combining an internally controlled real-time PCR assay for the detection of four protozoa in stool sample with a test algorithm for microscopy. Clin Microbiol Infect 15: 869-874.

39. Altschul SF, Gish W, Miller W, Myers EW, Lipman DJ (1990) Basic local alignment search tool. J Mol Biol 215: 403-410.

40. Bern C, Ortega Y, Checkley W, Roberts JM, Lescano AG, et al. (2002) Epidemiologic differences between cyclosporiasis and cryptosporidiosis in Peruvian children. Emerg Infect Dis 8: 581-585.

41. Bednarska M, Bajer A, Welc-Falęciak R, Pawełas A (2015) Cyclospora cayetanensis infection in transplant traveller: a case report of outbreak. Parasit \& Vectors 8: 411.

42. Fryauff DJ, Krippner R, Prodjodipuro P, Ewald C, Kawengian S, et al. (1999) Cyclospora cayetanensis among expatriate and indigenous populations of West Java, Indonesia. Emerg Infect Dis 5: 585-588.

43. Blans MCA, Ridwan BU, Verveij JJ, Rozenberg-Arska M, Verhoef J (2005) Cyclosporiasis outbreak, Indonesia. Emerg Infect Dis 11: 1453-1455. 